\section{MISIONES ACTUALES DE LAS UNIVERSIDADES PÚBLICAS: UNA PERSPECTIVA SOCIOLÓGICA}

\author{
Davinia Palomares Montero \\ Adela García Aracil \\ Elena Castro Martínez \\ INGENIO (CSIC-UPV) \\ Ciudad Politécnica de la Innovación. \\ Camino de Vera, s/n. Valencia (46022) Spain. \\ dpalomares@ingenio.upv.es \\ agarcia@ingenio.upv.es \\ ecastrom@ingenio.upv.es
}

\section{CURRENT MISSIONS OF PUBLIC UNIVERSITIES: A SOCIOLOGICAL PERSPECTIVE}

\begin{abstract}
From the New Sociological Institutionalism point of view, we have seen what the missions that institutionally Spanish public universities assume in their strategic plans are. It has been applied the content analysis approach. We have found that indicators are more frequent to teaching activities, in second place appeared research activities and in third place were knowledge transfer activities. Not all universities assume the same grade of institutionalization for each mission. In fact, we have obtained a typology of universities split in two groups, one focused to teaching and another focused to research and knowledge transfer.
\end{abstract}

KEY WORDS: New Sociological Institutionalism; University; Strategic Plan; University Missions.

\section{INTRODUCCIÓN}

A lo largo de la historia, la universidad ha cambiado sustancialmente los rasgos estructurales que la definen como institución, lo que se refleja especialmente en la definición de los fines que pretenden cumplir las organizaciones universitarias. Desde su origen en la Europa medieval hasta comienzos del siglo XIX, la universidad fue considerada sobre todo como un templo de sabiduría, donde la élite de especialistas producía y acumulaba conocimiento, normalmente en torno a tres ámbitos disciplinarios constituyentes del saber en la sociedad tradicional: la teología, el derecho, y la medicina (Martín, 2000). En los albores del siglo XIX, son las universidades alemanas las que contribuyen decisivamente al desarrollo de una misión que, junto a la enseñanza, empieza a ocupar una
RESUMEN: Este artículo analiza, desde la perspectiva del Nuevo Institucionalismo Sociológico, cuáles son las misiones que las universidades públicas españolas asumen de forma institucional en sus planes estratégicos. Para tal fin, se ha utilizado el análisis de contenido. En general, los resultados indican que las universidades muestran mayor énfasis en la misión "Docencia", seguida de la "Investigación", y en último lugar en la "Transferencia de conocimiento", si bien este patrón no es homogéneo en todas las universidades. Se han identificado dos tipologías de universidades en función del peso otorgado a cada misión: las más centradas en "Docencia" frente a las focalizadas en "Investigación y Transferencia de Conocimiento".

PALABRAS CLAVE: Nuevo Institucionalismo Sociológico; universidad; planificación estratégica; misiones universitarias.

gran importancia: la investigación científica. La visión de Wilhelm von Humboldt de la universidad como institución donde la investigación estaba unida estrechamente a la enseñanza, y dirigida especialmente al desarrollo social a través del cultivo de las ciencias, se extiende a lo largo del siglo XIX y la primera mitad del siglo XX en muchos países europeos y en Norteamérica; aunque generalmente la investigación universitaria se ha realizado con amplio grado de autonomía e independencia respecto al Estado y la empresa (Geuna, 1996). Durante la Segunda Guerra Mundial existe un importante punto de inflexión en los usos de la universidad, cuando sus capacidades científicas se ponen al servicio de la industria y el esfuerzo militar. Sin embargo, el rasgo definitorio de las décadas comprendidas entre 1950 y 1970 es la construcción de los estados del bienestar, lo que provoca una notable ex- 
pansión del acceso a la educación universitaria por parte de amplias capas de la población, con el consiguiente crecimiento de las universidades. Finalmente, el inicio de la revolución universitaria más reciente se encuentra en la década de los 80 , cuando el interés público comienza a reconocer más explícitamente el valor de la universidad en la economía del conocimiento (OCDE, 1999). Una de las manifestaciones de este cambio es la diferenciación de instituciones para responder a la demanda del mercado de enseñanza e investigación (Scott, 1998), siendo las universidades progresivamente más atractivas a las inversiones empresariales (Martín, 2000). Así, aparece un nuevo modelo de universidad que responde de forma creciente al cumplimiento de una "tercera misión", entendida como el "conjunto de actividades universitarias relacionadas con la generación de conocimiento y capacidades en colaboración con organizaciones y agentes no-académicos, así como con el uso, aplicación y explotación del conocimiento y otras capacidades existentes en la universidad, fuera del entorno académico" (Molas-Gallart y col., 2002).

En este contexto, el objetivo de este artículo es analizar el grado en que las universidades han interiorizado y adaptado sus misiones a las nuevas demandas del entorno, y cómo ello se plasma en las misiones universitarias y, como tal, aparece reflejado y legitimado en aquellos documentos que definen formalmente los objetivos que debe cumplir la universidad. Para ello utilizamos como caso estratégico de investigación el sistema universitario español. Nuestras unidades de análisis son cada una de las organizaciones universitarias en su conjunto. Aunque resulta complejo observar empíricamente algunos de los mecanismos de influencia, se considera especialmente útil revisar sus planes estratégicos, donde los gestores académicos hacen explícita cuál es la visión y la misión institucional. Ello, por tanto, nos permite comprender cómo los resultados pueden verse influidos por elementos simbólicos, tales como sus valores y misiones. De hecho, se puede considerar al plan estratégico como el principal mecanismo por el que se ejercen las influencias en el campo organizativo de la educación superior, dado que reflejan cómo reacciona la comunidad universitaria a las posibles presiones del entorno, principalmente legitimando sus valores y misiones para obtener el apoyo de los gobiernos y otras organizaciones de las que dependen para obtener recursos, especialmente la financiación. El análisis de estos documentos permitirá conocer, además, si las universidades públicas españolas planifican de forma homogénea el desarrollo de las tres misiones básicas de docencia, investigación y transferencia de conocimiento o si, por el contrario, existe un proceso de diferenciación entre ellas.

Para llevar a cabo este cometido, el artículo se organiza de la siguiente manera. En el punto 2, se expone un enfoque sociológico que resulta de utilidad para interpretar algunos de los cambios en las misiones universitarias. En el punto 3 , se describen los procesos de planificación estratégica como elemento relevante del cambio universitario. En el punto 4 , se expone la metodología del estudio empírico, especificando las fuentes de datos y la estrategia de análisis. En el punto 5, se exponen los resultados, divididos en dos apartados; en el primero de ellos se realiza un diagnóstico general del papel de las diversas misiones en la universidad española, y seguidamente se realiza una visión comparada centrada específicamente entre la docencia, la investigación y las actividades de tercera misión. En las conclusiones, presentadas en el punto 6 , se discuten las implicaciones de los resultados del estudio en lo referido a la orientación del sistema público español.

\section{El Nuevo Institucionalismo Sociológico COMO RECURSO PARA LA INTERPRETACIÓN DE LAS ORGANIZACIONES CIENTÍFICAS}

Entre la variada literatura que se ocupa de las organizaciones universitarias, el enfoque llamado Nuevo Institucionalismo Sociológico (NIS) resulta de utilidad para estudiar los procesos de transformación de las misiones institucionales por varias razones. En primer lugar, se adapta a las unidades de análisis disponibles cuando se pretende estudiar un sistema universitario en su conjunto. A saber, utiliza como unidad de análisis a las organizaciones, y las observa en el contexto de un campo organizativo (el entorno), lo cual es útil para observar posibles influencias y movimientos en el conjunto de dichas organizaciones. En segundo lugar, las dimensiones de observación del NIS no son sólo los resultados tipo outputs, sino los elementos simbólicos. Es decir, los valores y misiones. Esto es especialmente útil en el caso de organizaciones que producen resultados intangibles, tales como el conocimiento científico. Estos resultados pueden verse influidos especialmente por los sistemas de valores referidos a las tareas que se conside- 
ran más o menos legítimas en un determinado contexto. Adicionalmente, este enfoque supone una aproximación dinámica que es adecuada para observar el cambio de misiones. En tercer lugar, el NIS tiene algunas intuiciones que parecen acertadas en campos organizativos como el universitario, que dependen especialmente de las relaciones con el entorno, tanto en los recursos que reciben como en la orientación y regulación de sus actividades por parte de los poderes públicos.

Este enfoque presta atención a una serie de mecanismos, como por ejemplo el isomorfismo, que, si bien son difíciles de estudiar empíricamente, constituyen un marco de interpretación adecuado para analizar las observaciones empíricas sobre las misiones universitarias. A continuación, se exponen algunos rasgos de los autores más representativos, especialmente los que han estudiado a las universidades, debido a la relevancia de algunos conceptos para analizar los datos que se recogen en el caso del sistema universitario español.

En particular es interesante el programa de investigación de Meyer, desarrollado en el último cuarto del siglo XX. Este autor entiende a la ciencia no sólo como un instrumento, sino como una institución cultural que, en algunos de sus rasgos, afecta al resto de la sociedad. Desde este presupuesto, estudia la autoridad alcanzada por la institución científica, entendida como el elemento cultural primordial dotado de gran poder que define los modos de vida e impregna los sistemas de valores de la sociedad moderna globalizada (Meyer, 1977). El Neoinstitucionalismo defiende, por tanto, que la ciencia depende de las instituciones en las que se encarna, así como de otras fuerzas del entorno social.

Para estos autores, estos procesos funcionan, y por tanto se observan más adecuadamente, enraizados en organizaciones que están relacionadas entre ellas y con otras que forman parte de su entorno inmediato. En las sociedades modernas son las organizaciones las que realizan la coordinación imperativa de la acción social, movilizando recursos económicos, materiales y humanos para llevar a cabo objetivos concretos. En estas organizaciones la definición de objetivos es mutable, estando afectada por un proceso de construcción social donde funcionan una serie de mecanismos sociales que contribuyen a que una serie de fines se implanten frente a otros.
Uno de estos mecanismos es el llamado isomorfismo, entendido como un proceso que fuerza a una unidad perteneciente a una población, normalmente una organización ubicada en un campo organizativo relacionado, a parecerse a otras unidades que conviven en las mismas condiciones ambientales.

Meyer (1977) describe dos tipos de isomorfismo: el competitivo y el institucional. El primero enfatiza la competición de mercado, siendo relevante en contextos donde existen condiciones de libre mercado, aunque este modelo no suele reflejar adecuadamente a las organizaciones modernas. Por esta razón, el segundo tipo de isomorfismo, el institucional, hace referencia a la presión que producen otras organizaciones, ya que esto provoca una competición por ganar poder político y legitimación institucional. El proceso por el que determinados principios, políticas y prácticas, en este caso las referidas a la ciencia, se comienzan a implantar en algunas organizaciones, se observa prestando atención a los procesos de construcción social por los que una serie de prácticas comienzan a funcionar a modo de guiones cognitivos, dando lugar a la implantación de prácticas y modos de entender la realidad que terminan haciéndose predominantes (Meyer, 1977). Esto constituye un proceso que fuerza a miembros de la misma organización a aceptar prácticas consideradas legítimas, y a su vez a una unidad organizativa del total de la población a parecerse a otras unidades en su mismo campo organizativo (Hawley, 1968).

Meyer y Rowan (1977) utilizan el proceso de isomorfismo para explicar los fenómenos de las Ilamadas "organizaciones suaves" -sobre todo escuelas y universidades-. En este tipo de organizaciones, los criterios formales del desempeño económico no se aplican de manera directa ni se expresan de manera explícita en la estructura y las prácticas de la organización. En el caso de las universidades, señalan cómo, desde finales del siglo $X X$, se está modificando la relación de la universidad con las esferas de la producción y el mercado (Meyer, 2002; Meyer y Rowan, 2006). Es decir, nos referimos al impacto que están teniendo la privatización y comercialización de la educación y del conocimiento, que reconfigura el sistema de educación y ciencia como un sistema abierto, diversificado y complejo (Campbell, 2004). En este nuevo contexto, cobra relevancia el proceso de diversificación y segmentación del mercado de los servicios educativos, lo que modifica el significado

ARBOR Vol. 188753 enero-febrero [2012] 171-192 ISSN: 0210-1963

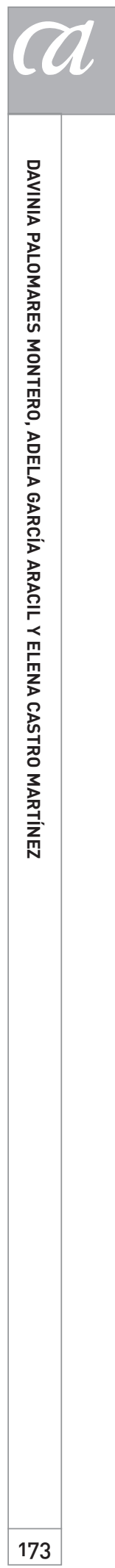

doi: 10.3989/arbor.2012.753n1011 
de la cultura y los símbolos en la construcción del sentido que los agentes otorgan a su realidad cotidiana (Meyer, 2006).

Por otra parte, el proceso de isomorfismo es analizado también por autores como DiMaggio y Powell acudiendo a una serie de factores más amplios. Éstos identifican tres mecanismos que explican su ocurrencia en las organizaciones: el coercitivo, el mimético y el normativo (DiMaggio y Powell, 1983). El primero, el coercitivo, proviene de la influencia política y de los problemas de legitimación. Las presiones que se ejercen en las instituciones por parte de otras organizaciones de las que se depende, persuaden o presionan para que éstas convivan con complicidad. Bajo estas circunstancias, el cambio organizacional es resultado de los mandatos gubernamentales, pues la existencia de normas legales comunes afecta, en muchos aspectos, a la estructura y comportamiento de la organización, haciendo que ambas sean progresivamente más homogéneas para alcanzar mayores cotas de legitimidad.

El segundo mecanismo, el proceso de mimetismo, surge de la aparición de respuestas estándar a situaciones de incertidumbre. En un contexto ambiguo, donde los objetivos no están claros, las organizaciones tienden a imitar a otras similares, consideradas como modelo ante situaciones complejas, con la intención de legitimar su comportamiento. Finalmente, el tercer mecanismo, el normativo, se asocia con la profesionalización, entendida ésta como la lucha colectiva de los miembros de una ocupación para definir las condiciones y los métodos de su trabajo y así legitimar su autonomía. Por tanto, el proceso de isomorfismo es un mecanismo que debe ser tenido en cuenta en el proceso de institucionalización de la ciencia, pues influye directamente en la forma en que la organización científica estructura, adapta o modifica sus dinámicas.

El terreno universitario es de nuevo un ámbito en el que las ideas de cambio organizativo resultan especialmente oportunas. Existen numerosas evidencias de que son varias las organizaciones que generan conocimiento científico y tecnológico que se ven afectadas por los cambios en la concepción de la ciencia (David y Foray, 1994). Las relaciones entre las instituciones generadoras de nuevo conocimiento y la sociedad son cada vez más complejas, estando regidas por la ambivalencia y la incertidumbre (Demeritt, 2000). Dado que la universidad es el principal lugar donde la institucionalización de la ciencia es más visible (Kerr, 1963; Geuna, 1996), y que se han convertido en las principales fábricas de conocimiento (Kerr, 1963; Geuna, 1996), son un ejemplo para observar la ocurrencia del isomorfismo institucional. Se puede decir que en ellas existe una brecha entre la estructura formal, centrada en el análisis de las exigencias que les plantea el contexto sociocultural para construir su legitimidad, y la consideración de su desempeño a partir de medidas asociadas a criterios de eficiencia y productividad. Las universidades son organizaciones complejas que a lo largo de la historia han ido acumulando funciones, como las de producción de saber, enseñanza y conocimiento científico. Pero además, recientemente son consideradas como pilares fundamentales en el desarrollo y la innovación basada en la ciencia (Godin y Gingras, 2000). En ellas se observa, por tanto, un proceso que hace emerger una serie de objetivos que aparecen cada vez más legitimados, cuyo principal exponente son los planes universitarios.

El empleo de este enfoque responde a la siguiente pregunta: ¿Cómo puede informar la interpretación de la evidencia disponible? ¿Es útil a la hora de sugerir posibles explicaciones a las orientaciones de las universidades españolas que se ven reflejadas en sus planes estratégicos? Es necesario reconocer que testar empíricamente algunos de sus postulados es especialmente difícil, principalmente los motivos por los que ocurre o no el isomorfismo organizativo. Para confirmar sus postulados sería necesario realizar un trabajo de carácter histórico, con secuencias de observaciones dilatadas en el tiempo que son difíciles de obtener. Además, es difícil observar empíricamente los mecanismos que operan, principalmente por las dificultades de conseguir datos estadísticos sobre el proceso. No obstante, el enfoque puede resultar de utilidad para hacer una interpretación. Más que un conjunto de hipótesis, se trata de una base teórica que proporciona una serie de supuestos útiles para interpretar las tendencias de cambio en un campo organizativo.

La implantación de las misiones universitarias de docencia, investigación y transferencia de conocimiento en el sistema español se tratarán de observar e interpretar en los siguientes apartados acudiendo a su plasmación en los procesos de planificación estratégica. 


\section{La PLANIFICACIÓN ESTRATÉGICA COMO MECANISMO DE INSTITUCIONALIZACIÓN DE LAS PRÁCTICAS UNIVERSITARIAS}

En las últimas décadas, las universidades están inmersas en una corriente continua de cambio, debido a múltiples factores; entre ellos: i) cambios demográficos, que producen que la demanda de educación superior presente importantes variaciones; ii) cambios socioeconómicos, que reclaman que la universidad se convierta en un factor clave de competitividad y calidad de vida de las regiones; iii) internacionalización de la educación y la ciencia, que ha dado lugar a la implantación del Espacio Europeo de Educación Superior (EEES) y del Espacio Europeo de Investigación (EEI); iv) proliferación de universidades, que aumenta la competencia por los alumnos, por los fondos para investigación y por el mejor personal; $y$, entre otros, v) cuestionamiento general de las bases de legitimación de las universidades, especialmente respecto de los servicios de las universidades públicas (Vilalta, 2001; Álamo Vera y García Soto, 2007).

Ante tales circunstancias, las universidades tratan de promover transformaciones en su organización para conseguir ser más competitivas, dinámicas y transparentes (Álamo Vera y García Soto, 2007; Sánchez y Elena, 2007). Sin embargo, esta reconfiguración de las universidades no es sencilla, por lo que comienza a ser necesaria la aplicación de herramientas de planificación que contribuyan a sistematizar y organizar las tareas a emprender.

En este sentido, en sus planes estratégicos las universidades describen lo que actualmente son y lo que desean ser. Del mismo modo, establecen qué se comprometen a ofrecer a los colectivos a los que dirigen sus servicios (Mintzberg et al., 1997; Arias Coello y Simón Martín, 2005), legitimando en su texto los valores y las misiones que debe perseguir la comunidad universitaria. No obstante, los planes estratégicos resultarian ser poco más que justificaciones de las actividades del pasado si a partir de ellos no se conceptualizan e implementan cambios significativos, con un fuerte apoyo de los dirigentes de la organización, pero también de toda la comunidad en su conjunto.

En el caso español, la planificación estratégica se introduce a mediados de la década de los noventa (Álamo Vera y García Soto, 2007), siendo poco después la Ley Orgánica de Universidades -LOU 2001- un factor impulsor de este mecanismo. Concretamente, el artículo 15, punto 1 de la LOU 2001 (que no se modificó en la LOU 2007) dictamina que el Consejo de Gobierno deberá establecer "las líneas estratégicas y programáticas de la Universidad, así como las directrices y procedimientos para su aplicación", por lo que podriamos pensar que el aumento del número de planes estratégicos en las universidades públicas españolas se debe, en parte, a un imperativo legal.

Sin embargo, la puesta en marcha de planes estratégicos en nuestras universidades no responde de forma exclusiva a un mecanismo coercitivo, sino también a otros de carácter mimético y normativo, tal y como ocurre en otros sistemas. De hecho, son diversos los trabajos realizados sobre las potencialidades de la planificación estratégica en instituciones de educación superior que reflejan procesos en este sentido. Por ejemplo, en Noruega se han analizado los planes estratégicos para conocer el valor añadido que tienen las reformas de la financiación en los resultados de investigación (Frølich y Klitkou, 2006) y en la India en los resultados académicos de los estudiantes (Bhattacharjee, 2008). Otros estudios tratan de proponer guías de buenas prácticas para desarrollar de forma adecuada planes estratégicos en algunos servicios ofertados, como por ejemplo en las bibliotecas (Fuentes Romero, 2001; Arias Coello y Simón Martín, 2005) o en algunas universidades españolas en general (Taylor y Machado, 2006; Álamo Vera y García Soto, 2007; Sánchez y Elena, 2007). El hecho es que esta práctica se ha extendido en las universidades públicas españolas, convirtiéndose en una de las herramientas utilizadas para definir la actuación futura de estas instituciones y trazar un plan de ruta con el cual guiar su rutina (Torres et al., 2008).

No debe olvidarse, sin embargo, que este instrumento de gestión, que ofrece una idea general sobre la que inferir cómo serán las universidades públicas españolas en un futuro no muy lejano, es un punto de partida sobre el que realizar nuestro análisis. Ofrece información útil y valiosa sobre el estado actual y futuro de la institución, y ayuda a sistematizar y organizar las tareas a emprender por parte de las distintas organizaciones del sistema (Álamo Vera y García Soto, 2007). Para el caso de nuestro análisis, estos planes se pueden interpretar como el principal mecanismo por el que se ejercen las influencias en este campo orga-

ARBOR Vol. 188753 enero-febrero [2012] 171-192 ISSN: 0210-1963 
nizativo, dado que reflejan cómo reacciona la comunidad universitaria a las posibles presiones del entorno, sobre todo a la legitimación que persiguen para obtener apoyo de los gobiernos regionales y otras organizaciones de las que dependen para obtener recursos. En suma, los planes estratégicos se pueden interpretar como un indicador de la legitimación de las misiones. En este sentido, la presencia de distintos elementos en estos documentos indica el papel de las misiones en el conjunto del sistema y en organizaciones concretas.

\section{Metodología}

\subsection{Fuentes de datos}

Para desarrollar nuestro trabajo empírico hemos realizado, en primer lugar, una búsqueda pormenorizada en las páginas web de las 47 universidades públicas españolas que ofrecen educación presencial, encontrando que 36 de estas universidades han definido y publicado su plan estratégico, lo que supone que el $77 \%$ del universo ha puesto en marcha esta herramienta de gestión. Se considera apropiada la selección de todos los casos. Por tanto, la muestra final está compuesta por los 36 planes estratégicos, correspondientes a la última edición publicada, de las universidades públicas españolas (véase Tabla 1).

No han sido incluidas en el estudio 11 universidades públicas españolas presenciales por las razones que se indican a continuación:

- La Universidad Complutense de Madrid, por la imposibilidad de obtener el documento de planificación estratégica.

- La Universidad Rey Juan Carlos y la Universidad de Extremadura tienen restringido el acceso al documento estratégico por medios electrónicos.

- Algunas universidades han desarrollado un plan estratégico para un área o servicio específico, como es el caso de la Universidad Pompeu Fabra, cuyo plan estratégico se centra exclusivamente en la investigación.

- La Universidad de Cantabria, la Universidad de Castilla La-Mancha y la Universidad Politécnica de Cartagena, que se centran en la gestión administrativa de la institución.
- La Universidad de Alcalá, la Universidad Carlos III y la Universidad Politécnica de Madrid, cuya planificación se dirige sólo al servicio de biblioteca de la universidad.

-El plan estratégico recientemente elaborado por la Universidad Autónoma de Barcelona va destinado a la planificación de lo que llaman "la universidad electrónica" y, por tanto, tiene objetivos distintos a los de la institución presencial, por lo que no es comparable con los demás.

Seis de los planes analizados son la renovación de una planificación anterior. Éste es el caso de la Universidad de Huelva, la Universidad de Málaga, la Universidad Miguel Hernández de Elche, la Universidad Politécnica de Cataluña, la Universidad Pablo de Olavide y la Universidad Pública de Navarra. Asimismo, nueve de los documentos analizados que se encuentran finalizados no han iniciado trámites para su renovación cuando se ha dado por concluida la búsqueda de planes estratégicos (8 de enero de 2010).

Resulta complejo averiguar cuáles son los motivos que explican las diferencias en cuanto al seguimiento y renovación de los planes estratégicos. Sin embargo, no podemos pasar por alto la situación de debate que se está viviendo en nuestro país en torno al desarrollo del anteproyecto de Ley de la Ciencia, la Tecnología y la Innovación. Analizando el borrador de dicho anteproyecto (Ministerio de Ciencia e Innovación, 2010), podemos observar que se modifican algunos artículos de la LOU 2001, entre ellos los que afectan al personal investigador y las actividades relacionadas con la transferencia de conocimiento, lo cual conlleva también modificaciones en los estatutos de las universidades. Podriamos pensar, por tanto, que algunas instituciones podrian esperar a desarrollar su plan estratégico hasta que la nueva Ley de la Ciencia fuera aprobada y, de esta forma, tras la actualización de sus estatutos, desarrollar su planificación.

Por otro lado, podemos observar que solo 26 de los 36 documentos analizados están en vigor, lo que supone un $72,2 \%$ de total. Es importante señalar que $17(65,4 \%)$ de los planes estratégicos que se encuentran en marcha han sido iniciados a partir del año 2007, fecha en la que se modificó la LOU 2001, si bien la totalidad de los planes estratégicos, a excepción del desarrollado por la Universidad Jaume I de Castellón, han sido elaborados después de la aprobación de la LOU 2001 (véase Tabla 1). 
TABLA 1. PLANES ESTRATÉGICOS DE LAS UNIVERSIDADES PÚBLICAS ESPAÑOLAS (ÚLTIMA EDICIÓN)

\begin{tabular}{|c|c|c|}
\hline Universidad & Período & Título \\
\hline A Coruña & $2005-10$ & Plano estratéxico de la UDC 2005-2010. Misión, Visión e Valores \\
\hline Alicante & $2007-12$ & Plan estratégico de la Universidad de Alicante (Horizonte 2012) \\
\hline Almería & $2003-06$ & Plan estratégico Universidad de Almería \\
\hline Autónoma de Madrid & $2003-06$ & Plan estratégico 2003-2006. Proyectos y acciones estratégicas \\
\hline Barcelona & $2008-20$ & Plan marco UB Horizonte 2020 \\
\hline Burgos & 2004-08 & Plan estratégico 2004-2008 \\
\hline Cádiz & $2005-10$ & Plan estratégico de la Universidad de Cádiz 2005-2010 \\
\hline Córdoba & $2006-15$ & Plan estratégico 2006-2015 \\
\hline Girona & 2008-13 & Pla estratègic 2008-2013 de la Universitat de Girona \\
\hline Granada & $2006-10$ & Plan estratégico $2006-2010$ \\
\hline Huelva & $2008-10$ & Plan estratégico institucional \\
\hline Islas Baleares & $2002-06$ & Pla estratègic de la Universitat de les Illes Balears, 2002-2006 \\
\hline Jaén & $2003-10$ & Plan estratégico 2003-2010 Universidad de Jaén \\
\hline Jaume I de Castellón & $2000-08$ & Sistema de dirección estratégica \\
\hline La Laguna & $2006-15$ & Plan estratégico de la Universidad de La Laguna \\
\hline La Rioja & $2002-05$ & Plan estratégico de la Universidad de La Rioja 2002-2005 \\
\hline Las Palmas de Gran Canaria & $2002-06$ & Plan estratégico institucional 2002-2006 \\
\hline León & $2006-08$ & Plan estratégico ULE 2milseis/2milocho \\
\hline Lleida & $2006-12$ & Pla estratègic 2006-2012. Conceptes centrals \\
\hline Málaga & $2009-12$ & Plan estratégico Universidad de Málaga 2009-2012 \\
\hline Miguel Hernández de Elche & $2008-12$ & III Plan de calidad. Universidad pública con excelencia acreditada \\
\hline Murcia & 2007-12 & Plan estratégico institucional 2007-2012 Universidad de Murcia \\
\hline Oviedo & $2005-10$ & La Universidad de Asturias. Plan estratégico 2005-2010 \\
\hline Pablo de Olavide & $2009-11$ & Plan estratégico de la UPO. 2009-2011 \\
\hline País Vasco & 2007-11 & Plan estratégico 2007-2011 de la UPV-EHU \\
\hline Politécnica de Catalunya & 2008-10 & Pla estratègic UPC \\
\hline Politécnica de Valencia & $2007-14$ & Plan estratégico UPV. 2007-2014 \\
\hline Pública de Navarra & $2005-08$ & Universidad Pública de Navarra. II Plan estratégico. 2005-2008 \\
\hline Rovira i Virgili & $2008-13$ & Pla Estratègic \\
\hline Salamanca & $2009-12$ & Plan Estratégico USAC 2009-2012 \\
\hline Santiago de Compostela & $2003-10$ & Plan estratégico de la USC. Objetivos estratégicos \\
\hline Sevilla & $2008-12$ & Plan estratégico Universidad de Sevilla \\
\hline Valencia Estudi General & $2008-11$ & Plan estratégico 2008-2011 \\
\hline Valladolid & $2008-14$ & Plan estratégico de la Universidad de Valladolid 2008-2014 \\
\hline Vigo & $2008-12$ & Plan estratégico formato electrónico 2008-2012 \\
\hline Zaragoza & $2002-05$ & Plan estratégico 2002-2005 \\
\hline
\end{tabular}

Fuente: Elaboración propia. 


\subsection{Estrategia de análisis}

En el presente artículo se ha aplicado el método de análisis de contenido por su capacidad para extraer un conocimiento en profundidad de los valores presentes en los mensajes, textos o discursos (Colyvas y Powell, 2006).

Desarrollar correctamente el método conlleva definir las unidades de análisis, que son los elementos sobre los que se centra el estudio y que se dividen en: unidades de muestreo (unidades materiales que conforman la realidad a investigar), de contexto (unidades amplias que contienen la información referida en la unidad de registro) y de registro (segmentos de contenido que pueden ser categorizados, medidos, descritos, analizados e interpretados). En el caso que nos ocupa, las "Unidades de Muestreo" son los planes estratégicos de las 36 universidades públicas españolas presenciales. Como "Unidades de Contexto" se han establecido las partes en las que generalmente se estructuran los planes estratégicos, es decir, la "Misión" (propósito, funciones y valores de la organización), la "Visión" (lo que la organización desea ser) y los "Ejes" (objetivos y estrategias de actuación). Finalmente, las "Unidades de Registro" son la "Docencia" (entendida como "la transmisión de conocimientos y destrezas a los estudiantes a través de la formación y de los procesos de capacitación"), la "Investigación" (considerada como la "generación de nuevo conocimiento a través del análisis y la experimentación") y la "Transferencia de conocimiento" (entendida como el "establecimiento de vínculos con otros ámbitos no académicos a través de la transferencia de las capacidades universitarias y sus resultados y/o la comercialización de los recursos tecnológicos y científicos") (Molas-Gallart y col., 2002, op. cit.; Göransson et al., 2009). La Figura 1 muestra el planteamiento teórico-práctico diseñado para realizar el análisis de contenido de dichos planes estratégicos.

Definidas las unidades de análisis, se realiza una integración de los documentos a partir de un proceso de categorización, donde se organizan y clasifican las unidades de registro sobre la base de criterios de analogía y diferenciación. Se trata de un proceso de transformación de los datos brutos, los planes estratégicos, en datos útiles donde se subraya y marca el material original. De esta forma, se obtiene un corpus del material, señalado con diferentes medios, y acotado con proposiciones y conceptos que han emergido durante el proceso, permitiendo formular una lista de indicadores para presentar el marco teórico que define cada unidad de registro (véase Tabla 2). Ésta es, quizás, la etapa en la que los conocimientos de los autores desempeñan un papel importante, ya que ha sido necesario reducir el número de indicadores en base a criterios de equivalencia y similitud para poder obtener un listado final manejable estadísticamente.

FIGURA 1. MARCO TEÓRICO-PRÁCTICO DEL ANÁLISIS DE CONTENIDO DE LOS PLANES ESTRATÉGICOS

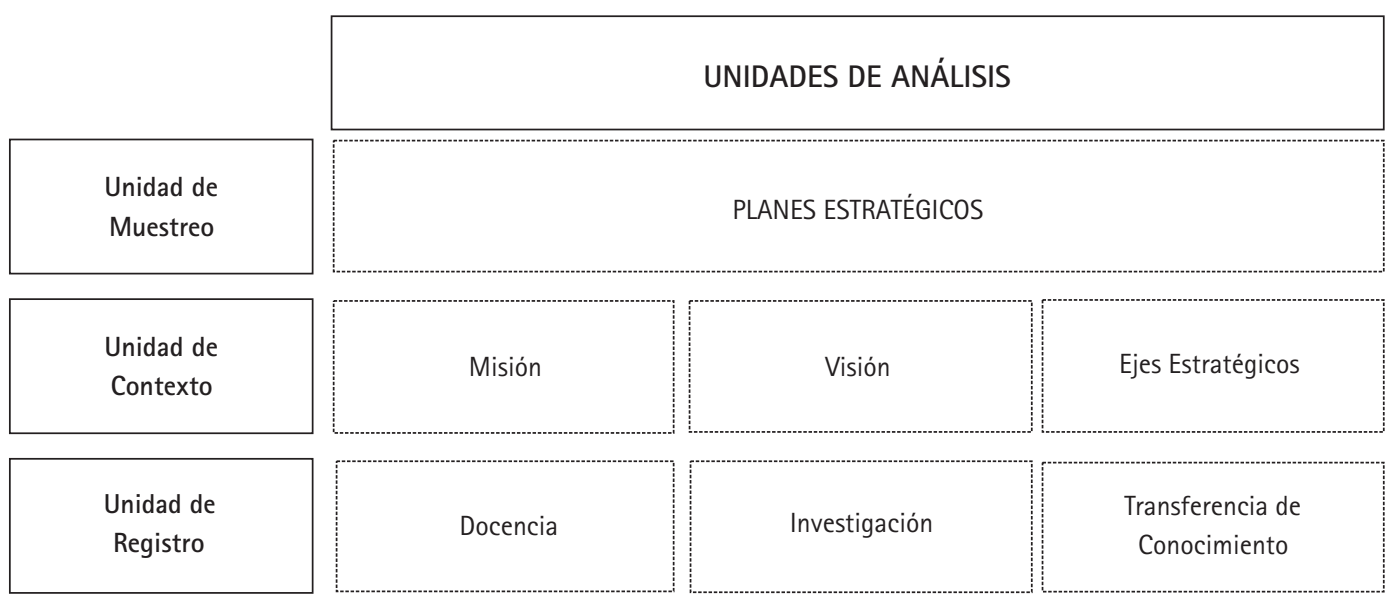

Fuente: Elaboración propia. 
Tal como se observa en la Tabla 2, la lista propuesta está compuesta por dos niveles para cada unidad de registro; por un lado los indicadores, que son la unidad de significación más elemental de información que se produce en el proceso de construcción del conocimiento y, por otro, las dimensiones, que representan constructos más generales como expresión de la interpretación y comparación de los indicadores definidos.

TABLA 2. LISTA DE INDICADORES EXTRAÍDA DE LOS PLANES ESTRATÉGICOS

\begin{tabular}{|c|c|c|}
\hline Unidad de registro & Dimensión & Indicadores \\
\hline \multirow{2}{*}{ Docencia } & Recursos y actividades & $\begin{array}{l}\text { Becas a estudios } \\
\text { Carga docente, dedicación docente } \\
\text { Enseñanza virtual, a distancia, e-learning } \\
\text { Estudiantes } \\
\text { Estudios de grado } \\
\text { Evaluación de la docencia } \\
\text { Formación continua, a lo largo de la vida } \\
\text { Infraestructuras académicas } \\
\text { Metodología docente, métodos didácticos } \\
\text { Movilidad (estudiantes y profesores) } \\
\text { NTIC en la docencia } \\
\text { Oferta académica } \\
\text { Profesor, profesorado, docente, académico, tutor } \\
\text { Titulaciones }\end{array}$ \\
\hline & Resultados e impactos & $\begin{array}{l}\text { Acreditación } \\
\text { Aprendizaje } \\
\text { Búsqueda empleo, ocupación, mercado laboral } \\
\text { Calidad de la docencia } \\
\text { Competencia profesional } \\
\text { Ejercicio profesional } \\
\text { Formación integral } \\
\text { Inserción laboral, empleabilidad } \\
\text { Internacionalización } \\
\text { Multidisciplinariedad } \\
\text { Premios al rendimiento académico } \\
\text { Preparación para la profesión, formación profesional } \\
\text { Rendimiento académico }\end{array}$ \\
\hline \multirow[t]{2}{*}{ Investigación } & Recursos y actividades & $\begin{array}{l}\text { Año sabático para investigación } \\
\text { Becas a la investigación } \\
\text { Carga investigadora, dedicación a investigación } \\
\text { Curso introducción/iniciación a la investigación } \\
\text { Doctorado, Doctorado de excelencia } \\
\text { Estudios de postgrado } \\
\text { Evaluación de la investigación } \\
\text { Fomento/apoyo a la investigación } \\
\text { Grupos de investigación } \\
\text { Infraestructura para la investigación } \\
\text { Máster } \\
\text { Personal investigador } \\
\text { Prácticas en actividad investigadora } \\
\text { Proyectos de investigación }\end{array}$ \\
\hline & Resultados e impactos & $\begin{array}{l}\text { Cooperación } \\
\text { Creación, producción, generación de conocimiento } \\
\text { Creatividad } \\
\text { Doctores } \\
\text { Investigación de excelencia (calidad) } \\
\text { Progreso, avance científico } \\
\text { Publicaciones } \\
\text { Sexenios, reconocimientos } \\
\text { Tesis doctorales }\end{array}$ \\
\hline
\end{tabular}


TABLA 2. LISTA DE INDICADORES EXTRAIIDA DE LOS PLANES ESTRATÉGICOS (continuación)

\begin{tabular}{|c|c|c|}
\hline Unidad de registro & Dimensión & Indicadores \\
\hline \multirow{2}{*}{$\begin{array}{c}\text { Transferencia } \\
\text { de conocimiento }\end{array}$} & Recursos y actividades & $\begin{array}{l}\text { Captación, búsqueda de recursos y fondos } \\
\text { Cátedras de empresa } \\
\text { Consejo Social } \\
\text { Consorcios } \\
\text { Consultoria, asesoramiento } \\
\text { Contratos y convenios } \\
\text { Desarrollo tecnológico } \\
\text { Divulgación científica } \\
\text { Emprendimiento } \\
\text { Incubadora de empresas } \\
\text { Intercambio de profesionales } \\
\text { OTRI } \\
\text { Parque científico } \\
\text { Prácticas en empresa }\end{array}$ \\
\hline & Resultados e impactos & $\begin{array}{l}\text { Creación de empresas } \\
\text { Diálogo social } \\
\text { Explotación comercial, comercialización } \\
\text { Implicación, compromiso social } \\
\text { Innovación } \\
\text { Licencias } \\
\text { Modelos de utilidad } \\
\text { Patentes } \\
\text { Relación con entorno empresarial y productivo } \\
\text { Servicio a la sociedad } \\
\text { Spin-off } \\
\text { Start-up } \\
\text { Transferencia tecnológica } \\
\text { Utilidad social }\end{array}$ \\
\hline
\end{tabular}

Fuente: Elaboración propia.

La lectura de estos indicadores nos ofrece una información exhaustiva acerca de las misiones de la universidad. Esta primera aproximación cualitativa al léxico utilizado en los planes estratégicos permite no sólo la descripción del contenido sino la interpretación amplia de las misiones de la universidad.

\section{Resultados}

\subsection{Visión general de las misiones de la universidad pública española}

El propósito de esta sección es ofrecer una visión general de las misiones asumidas por las universidades públicas españolas, que se desprende del análisis de la visión, la misión y los ejes estratégicos que éstas manifiestan en su planificación estratégica institucional, entendiendo que esta forma de gestión es su manera de planificar la actuación futura y, por tanto, nos informa de cuál es su ethos (del griego ethe, su personalidad).

La Tabla 3 presenta, para el total de la muestra objeto de estudio, la distribución de la aparición de los indicadores registrados por unidad de contexto (Misión, Visión y Ejes). Tras la revisión de los documentos se ha contabilizado un total de 17.573 apariciones de los indicadores. Cerca del 96\% han sido propuestos en la unidad de contexto "Ejes", mientras que sólo el 2\% aparece en la unidad "Misión" y el mismo porcentaje en la unidad "Visión". Esta distribución tiene sentido, si se tiene en cuenta que el número de páginas dedicadas a abordar la Misión y la Visión de la institución es considerablemente menor que el dedicado a plantear, explicar y desarrollar los Ejes, que recogen las estrategias de actuación para alcanzar la misión y visión. 
TABLA 3. NÚMERO TOTAL Y DISTRIBUCIÓN PORCENTUAL DE LA APARICIÓN DE INDICADORES POR UNIDAD DE CONTEXTO

\begin{tabular}{|c|c|c|c|c|c|c|c|c|}
\hline \multirow{3}{*}{ Unidad de Muestreo } & \multicolumn{6}{|c|}{ Unidad de Contexto } & \multirow{2}{*}{\multicolumn{2}{|c|}{ Total }} \\
\hline & \multicolumn{2}{|c|}{ Misión } & \multicolumn{2}{|c|}{ Visión } & \multicolumn{2}{|c|}{ Ejes } & & \\
\hline & N. ${ }^{\circ}$ & $\%$ & N. ${ }^{\circ}$ & $\%$ & $\mathrm{~N} .{ }^{\circ}$ & $\%$ & N. ${ }^{\circ}$ & $\%$ \\
\hline Total Indicadores & 355 & 2,0 & 379 & 2,2 & 16.839 & 95,8 & 17.573 & 100,0 \\
\hline
\end{tabular}

Fuente: Elaboración propia.

La Tabla 4, recoge la frecuencia de los indicadores por unidad de contexto y unidad de registro para el total de la muestra. Por término medio, el 51,5\% de los indicadores que aparecen son relativos a la "Docencia", el 25,8\% a la "Investigación" y el 22,7\% a la "Transferencia de conocimiento".

Centrando la atención en la unidad de contexto "Misión" se puede observar que el $47,0 \%$ de los indicadores hacen referencia a la "Docencia", el 32,7\% a la "Transferencia de conocimiento" y el 20,3\% de los indicadores contabilizados hacen referencia a la "Investigación". Este comportamiento se percibe también en la unidad de contexto "Visión", si bien, en este caso, el porcentaje de indicadores que hacen referencia a "Investigación" y "Transferencia de conoci- miento" son más próximos $(23,0 \%$ y $24,0 \%$, respectivamente). Finalmente, en la unidad de contexto "Ejes", donde se encuentra la gran parte de los indicadores computados, se observa que el porcentaje de indicadores con relación a la "Investigación" (26,0\%) es ligeramente mayor que el que aparece para la "Transferencia de conocimiento" $(22,5 \%)$. Por tanto, podríamos decir que la mayor tradición de nuestras universidades por la docencia queda reflejada en sus planes estratégicos ya que, de forma general, están dedicando mayor atención a esta misión en comparación con las otras dos. Asimismo, no es de extrañar que en la "Misión" y la "Visión" de las universidades aparezcan con mayor frecuencia indicadores relativos a la "Transferencia de conocimiento" ya que se tiende a hacer más hincapié en los aspectos menos desarrollados.

TABLA 4. NÚMERO TOTAL Y DISTRIBUCIÓN PORCENTUAL DE LA APARICIÓN DE INDICADORES POR UNIDAD DE CONTEXTO Y UNIDAD DE REGISTRO

\begin{tabular}{|c|c|c|c|c|c|c|c|c|}
\hline \multirow{3}{*}{$\begin{array}{l}\text { Unidad de } \\
\text { Contexto }\end{array}$} & \multicolumn{6}{|c|}{ Unidad de Registro } & \multirow{2}{*}{\multicolumn{2}{|c|}{ Total }} \\
\hline & \multicolumn{2}{|c|}{ Docencia } & \multicolumn{2}{|c|}{ Investigación } & \multicolumn{2}{|c|}{ Transferencia de conocimiento } & & \\
\hline & N. ${ }^{\circ}$ & $\%$ & N. ${ }^{\circ}$ & $\%$ & N. ${ }^{\circ}$ & $\%$ & N. ${ }^{\circ}$ & $\%$ \\
\hline Misión & 167 & 47,0 & 72 & 20,3 & 116 & 32,7 & 355 & 100,0 \\
\hline Visión & 201 & 53,0 & 87 & 23,0 & 91 & 24,0 & 379 & 100,0 \\
\hline Ejes & 8.684 & 51,6 & 4.370 & 26,0 & 3.785 & 22,5 & 16.839 & 100,0 \\
\hline Total & 9.052 & 51,5 & 4.529 & 25,8 & 3.992 & 22,7 & 17.573 & 100,0 \\
\hline
\end{tabular}

Fuente: Elaboración propia.

Por su parte, la Tabla 5 proporciona información más detallada, mediante la distribución de los indicadores para cada una de las misiones (unidades de registro) y sus correspondientes dimensiones, "Recursos y Actividades" y "Resultados e Impactos", lo cual nos proporciona testimonio de cómo son conceptualizadas las distintas misiones 
TABLA 5. NÚMERO TOTAL Y DISTRIBUCIÓN PORCENTUAL DE LA APARICIÓN DE INDICADORES POR UNIDAD DE REGISTRO Y DIMENSIONES

\begin{tabular}{|c|c|c|c|c|c|c|}
\hline \multirow{3}{*}{ Unidad de Registro } & \multicolumn{4}{|c|}{ Dimensiones } & \multirow{2}{*}{\multicolumn{2}{|c|}{ Total }} \\
\hline & \multicolumn{2}{|c|}{ Recursos/Actividades } & \multicolumn{2}{|c|}{ Resultados/Impactos } & & \\
\hline & N. ${ }^{\circ}$ & $\%$ & N. ${ }^{\circ}$ & $\%$ & N. ${ }^{\circ}$ & $\%$ \\
\hline Docencia & 6.386 & 70,5 & 2.666 & 29,5 & 9.052 & 100,0 \\
\hline Investigación & 3.201 & 70,7 & 1.328 & 29,3 & 4.529 & 100,0 \\
\hline Transferencia de conocimiento & 1.697 & 42,5 & 2.295 & 57,5 & 3.992 & 100,0 \\
\hline Total & 11.284 & 64,2 & 6.289 & 35,8 & 17.573 & 100,0 \\
\hline
\end{tabular}

Fuente: Elaboración propia.

en las universidades españolas. De forma global puede verse cómo los indicadores registrados hacen referencia, en primer lugar, a los "Recursos y Actividades" (64,2\%) y, con una menor frecuencia, a los "Resultados e Impactos" $(35,8 \%)$. Este resultado es curioso si pensamos que los ejercicios de planificación están dirigidos a la consecución de los objetivos marcados. Podríamos pensar, por tanto, que los planes estratégicos de las universidades públicas españolas están siendo utilizados principalmente como una herramienta de gestión más orientada al uso de los recursos que a la obtención de resultados.

Sin embargo, la distribución de los indicadores en cada dimensión no presenta la misma tendencia en todas las unidades de registro. Los indicadores contabilizados en la misión "Docencia" están mayoritariamente relacionados con los "Recursos y Actividades" (70,5\%), mientras que sólo el 29,5\% de los indicadores hacen referencia directa y explícita a los "Resultados e Impactos" esperados como consecuencia de la docencia, como es el propio proceso de aprendizaje, la calidad de la docencia o la inserción laboral de los graduados, entre otros. En cuanto a la misión "Investigación" se observa que también cerca de las tres cuartas partes de los indicadores se refieren a los "Recursos y Actividades" dedicados a "Investigación", mientras que el resto (29,3\%) figuran en la dimensión "Resultados e Impactos". En cambio, en la misión "Transferencia de conocimiento" más de la mitad de los indicadores contabilizados se refieren a los "Resultados e Impactos" obtenidos por desarrollar esta misión (57,5\%). Las diferencias detectadas entre las misiones no es una cuestión trivial. Las causas pueden ser varias, si bien podríamos pensar que, en cuanto a la docencia y la investigación, al ser misiones ampliamente conocidas por la comunidad académica, los gestores tratan de profundizar en los aspectos que deben trabajar los académicos, mientras que en la transferencia de conocimiento, al ser una misión de incorporación más reciente, los gestores tratan de marcar hacia dónde deben dirigirse los trabajos, siendo necesario un mayor esfuerzo conceptual para establecer los resultados e impactos principales que se deben alcanzar.

Por su parte, la Tabla 6 muestra la información anterior distribuida, además, por unidad de contexto. Para las tres misiones básicas de las universidades, la mayor parte de los indicadores contabilizados en la "Misión" se orientan principalmente a la dimensión "Resultados e Impactos": 53,9\% para "Docencia", 59,7\% en la "Investigación" e incluso mayor en la "Transferencia de conocimiento", 82,8\%. Éste es un resultado bastante lógico, puesto que la misión expresa lo que se pretende lograr.

Si nos centramos en la unidad de contexto "Visión", podemos ver que más de la mitad de los indicadores contabilizados se refieren a los "Recursos y Actividades", para el caso de la "Docencia" $(54,7 \%)$ y de la "Investigación" $(59,8 \%)$, no siendo así en la "Transferencia de conocimiento" que recoge un porcentaje muy inferior $(15,4 \%)$ que podría deberse a la falta de conocimiento y experiencia acerca de cuáles son los mecanismos que se pueden poner en práctica. En el caso de los "Ejes", la diferencia entre los indicadores registrados en ambas dimensiones es más pronunciada para el caso de la "Docencia" e "Investigación" (71,4\% en "Recursos y Actividades" frente a 
TABLA 6. NÚMERO TOTAL Y DISTRIBUCIÓN PORCENTUAL DE LA APARICIÓN DE INDICADORES POR UNIDAD DE REGISTRO, UNIDAD DE CONTEXTO Y DIMENSIONES

\begin{tabular}{|c|c|c|c|c|c|c|c|}
\hline \multirow{3}{*}{$\begin{array}{l}\text { Unidad de } \\
\text { Registro }\end{array}$} & \multirow{3}{*}{$\begin{array}{l}\text { Unidad de } \\
\text { Contexto }\end{array}$} & \multicolumn{4}{|c|}{ Dimensiones } & \multirow{2}{*}{\multicolumn{2}{|c|}{ Total }} \\
\hline & & \multicolumn{2}{|c|}{ Recursos/Actividades } & \multicolumn{2}{|c|}{ Resultados/Impactos } & & \\
\hline & & N. ${ }^{\circ}$ & $\%$ & N. ${ }^{\circ}$ & $\%$ & $\mathrm{~N} .{ }^{\circ}$ & $\%$ \\
\hline \multirow{3}{*}{ Docencia } & Misión & 77 & 46,1 & 90 & 53,9 & 167 & 100,0 \\
\hline & Visión & 110 & 54,7 & 91 & 45,3 & 201 & 100,0 \\
\hline & Ejes & 6.199 & 71,4 & 2.485 & 28,6 & 8.684 & 100,0 \\
\hline \multirow{3}{*}{ Investigación } & Misión & 29 & 40,3 & 43 & 59,7 & 72 & 100,0 \\
\hline & Visión & 52 & 59,8 & 35 & 40,2 & 87 & 100,0 \\
\hline & Ejes & 3.120 & 71,4 & 1.250 & 28,6 & 4.370 & 100,0 \\
\hline \multirow{3}{*}{$\begin{array}{c}\text { Transferencia } \\
\text { de conocimiento }\end{array}$} & Misión & 20 & 17,2 & 96 & 82,8 & 116 & 100,0 \\
\hline & Visión & 14 & 15,4 & 77 & 84,6 & 91 & 100,0 \\
\hline & Ejes & 1.663 & 43,9 & 2.122 & 56,1 & 3.785 & 100,0 \\
\hline
\end{tabular}

Fuente: Elaboración propia.

$28,6 \%$ en "Resultados e Impactos"), siendo más suavizada esta diferencia entre dimensiones en la "Transferencia de conocimiento" (43,9\% en "Recursos y Actividades" frente a $56,1 \%$ en "Recursos e Impactos"). Se trata de un resultado esperado especialmente si pensamos en la labor realizada por la Red OTRI, a través de su Grupo de Trabajo de Indicadores, que se ha encargado, en los últimos años, de desarrollar e implementar herramientas y actividades de medición de la actividad de transferencia de conocimiento y tecnología que realizan las universidades españolas.

Finalmente, la Tabla 7 ofrece la frecuencia y distribución porcentual de aparición de cada indicador por unidad de registro y por dimensión. En el caso de la unidad de registro "Docencia", para la dimensión "Recursos y Actividades", los cuatro indicadores con mayor presencia son los estudiantes $(20,8 \%)$, seguido de las titulaciones $(15,3 \%)$, los profesores $(15,0 \%)$ y la oferta académica $(10,9 \%)$, ya que juntos representan más del $60 \%$ en dicha dimensión. En "Resultados e Impactos" aparecen como importantes, en general, la calidad de la docencia y su repercusión en el mundo laboral (véase calidad de docencia $(20,6 \%)$, internacionalización $(19,8 \%)$, aprendizaje $(15,9 \%)$ y em- pleabilidad ( $8,0 \%)$, entre otros). Por tanto, en esta unidad de registro, los planes estratégicos están revelando mayor interés en indicadores relativos a alumnos, personal docente, oferta educativa y la adecuación de los objetivos del curso a la realidad profesional, a través de la calidad de la docencia y la empleabilidad de los graduados. Sin embargo, parece ser que no se preocupan tanto por la motivación de los profesores por enseñar, ni por la movilidad de los estudiantes, así como tampoco por el papel que debe desempeñar la universidad en la formación de emprendedores, lo que no refleja una asunción de objetivos sociales por las universidades, pues la situación socioeconómica hace que, al igual que otras entidades sociales, se demande a la institución universitaria que sea motor para lograr el cambio de modelo económico y social en nuestro país.

En el caso de la unidad de registro "Investigación", los indicadores más frecuentes en "Recursos y Actividades" son: el personal investigador (18,8\%), los estudios de postgrado $(14,2 \%)$, el año sabático para la investigación $(13,2 \%)$ y los proyectos de investigación (11,1\%). Para la dimensión "Resultados e Impactos", los indicadores con mayor presencia en los planes estratégicos son los relativos a la cooperación

ARBOR Vol. 188753 enero-febrero [2012] 171-192 ISSN: 0210-1963 
TABLA 7. FRECUENCIA Y DISTRIBUCIÓN PORCENTUAL DE CADA INDICADOR POR UNIDAD DE REGISTRO Y DIMENSIÓN

\begin{tabular}{|c|c|c|c|c|c|}
\hline $\begin{array}{l}\text { Unidad } \\
\text { de registro }\end{array}$ & Dimensión & Indicadores & $\begin{array}{c}\mathrm{N} .{ }^{\circ} \\
\text { absoluto }\end{array}$ & $\begin{array}{c}\% \\
(d=100)\end{array}$ & $\begin{array}{c}\% \\
\text { (u. reg=100) }\end{array}$ \\
\hline \multirow{29}{*}{ Docencia } & \multirow{14}{*}{$\begin{array}{l}\text { Recursos y } \\
\text { actividades }\end{array}$} & Estudiantes & 1.327 & 20,8 & 14,7 \\
\hline & & Titulaciones & 978 & 15,3 & 10,8 \\
\hline & & Profesor, profesorado, docente, académico, tutor & 957 & 15,0 & 10,6 \\
\hline & & Oferta académica & 697 & 10,9 & 7,7 \\
\hline & & NTIC en la docencia & 326 & 5,1 & 3,6 \\
\hline & & Metodologia docente, métodos didácticos & 318 & 5,0 & 3,5 \\
\hline & & Evaluación de la docencia & 316 & 4,9 & 3,5 \\
\hline & & Movilidad (estudiantes y profesores) & 308 & 4,8 & 3,4 \\
\hline & & Formación continua, a lo largo de la vida & 295 & 4,6 & 3,3 \\
\hline & & Estudios de grado & 288 & 4,5 & 3,2 \\
\hline & & Infraestructuras académicas & 196 & 3,1 & 2,2 \\
\hline & & Enseñanza virtual, a distancia, e-learning & 187 & 2,9 & 2,1 \\
\hline & & Carga docente, dedicación docente & 123 & 1,9 & 1,4 \\
\hline & & Becas a estudios & 70 & 1,1 & 0,8 \\
\hline & \multicolumn{2}{|c|}{ Total recursos y actividades } & 6.386 & 100,0 & 70,5 \\
\hline & \multirow{13}{*}{$\begin{array}{l}\text { Resultados e } \\
\text { impactos }\end{array}$} & Calidad de la docencia & 548 & 20,6 & 6,1 \\
\hline & & Internacionalización & 529 & 19,8 & 5,8 \\
\hline & & Aprendizaje & 423 & 15,9 & 4,7 \\
\hline & & Inserción laboral, empleabilidad & 214 & 8,0 & 2,4 \\
\hline & & Preparación para la profesión, formación profesional & 191 & 7,2 & 2,1 \\
\hline & & Formación integral & 149 & 5,6 & 1,6 \\
\hline & & Búsqueda empleo, ocupación, mercado laboral & 132 & 5,0 & 1,5 \\
\hline & & Acreditación & 115 & 4,3 & 1,3 \\
\hline & & Competencia profesional & 102 & 3,8 & 1,1 \\
\hline & & Rendimiento académico & 99 & 3,7 & 1,1 \\
\hline & & Premios al rendimiento académico & 81 & 3,0 & 0,9 \\
\hline & & Ejercicio profesional & 43 & 1,6 & 0,5 \\
\hline & & Multidisciplinariedad & 40 & 1,5 & 0,4 \\
\hline & \multicolumn{2}{|c|}{ Total resultados e impactos } & 2.666 & 100,0 & 29,5 \\
\hline \multicolumn{3}{|l|}{ Total docencia } & 9.052 & 100,0 & 100,0 \\
\hline
\end{tabular}


TABLA 7. FRECUENCIA Y DISTRIBUCIÓN PORCENTUAL DE CADA INDICADOR POR UNIDAD DE REGISTRO Y DIMENSIÓN (continuación)

\begin{tabular}{|c|c|c|c|c|c|}
\hline $\begin{array}{l}\text { Unidad } \\
\text { de registro }\end{array}$ & Dimensión & Indicadores & $\begin{array}{c}\mathrm{N} .{ }^{\circ} \\
\text { absoluto }\end{array}$ & $\begin{array}{c}\% \\
(d=100)\end{array}$ & $\begin{array}{c}\% \\
\text { (u. reg }=100)\end{array}$ \\
\hline \multirow{25}{*}{ Investigación } & \multirow{14}{*}{$\begin{array}{l}\text { Recursos y } \\
\text { actividades }\end{array}$} & Personal investigador & 601 & 18,8 & 13,3 \\
\hline & & Estudios de postgrado & 453 & 14,2 & 10,0 \\
\hline & & Año sabático para investigación & 421 & 13,2 & 9,3 \\
\hline & & Proyectos de investigación & 355 & 11,1 & 7,8 \\
\hline & & Grupos de investigación & 321 & 10,0 & 7,1 \\
\hline & & Evaluación de la investigación & 245 & 7,7 & 5,4 \\
\hline & & Infraestructura para la investigación & 233 & 7,3 & 5,1 \\
\hline & & Doctorado, Doctorado de excelencia & 216 & 6,7 & 4,8 \\
\hline & & Master & 136 & 4,2 & 3,0 \\
\hline & & Fomento/Apoyo a la investigación & 87 & 2,7 & 1,9 \\
\hline & & Becas a la investigación & 68 & 2,1 & 1,5 \\
\hline & & Carga investigadora, dedicación a investigación & 38 & 1,2 & 0,8 \\
\hline & & Curso introducción/iniciación a la investigación & 21 & 0,7 & 0,5 \\
\hline & & Prácticas en actividad investigadora & 6 & 0,2 & 0,1 \\
\hline & \multicolumn{2}{|c|}{ Total recursos y actividades } & 3.201 & 100,0 & 70,7 \\
\hline & \multirow{9}{*}{$\begin{array}{l}\text { Resultados e } \\
\text { impactos }\end{array}$} & Cooperación & 354 & 26,7 & 7,8 \\
\hline & & Creación, producción, generación de conocimiento & 296 & 22,3 & 6,5 \\
\hline & & Publicaciones & 215 & 16,2 & 4,7 \\
\hline & & Doctores & 135 & 10,2 & 3,0 \\
\hline & & Tesis doctorales & 98 & 7,4 & 2,2 \\
\hline & & Progreso, avance científico & 95 & 7,2 & 2,1 \\
\hline & & Creatividad & 52 & 3,9 & 1,1 \\
\hline & & Investigación de excelencia (calidad) & 45 & 3,4 & 1,0 \\
\hline & & Sexenios, reconocimientos & 38 & 2,9 & 0,8 \\
\hline & \multicolumn{2}{|c|}{ Total resultados e impactos } & 1.328 & 100,0 & 29,3 \\
\hline \multicolumn{3}{|c|}{ Total investigación } & 4.529 & 100,0 & 100,0 \\
\hline
\end{tabular}


TABLA 7. FRECUENCIA Y DISTRIBUCIÓN PORCENTUAL DE CADA INDICADOR POR UNIDAD DE REGISTRO Y DIMENSIÓN (continuación)

\begin{tabular}{|c|c|c|c|c|c|}
\hline $\begin{array}{l}\text { Unidad } \\
\text { de registro }\end{array}$ & Dimensión & Indicadores & $\begin{array}{c}\mathrm{N}^{\circ}{ }^{\circ} \\
\text { absoluto }\end{array}$ & $\begin{array}{c}\% \\
(d=100)\end{array}$ & $\begin{array}{c}\% \\
\text { (u. reg=100) }\end{array}$ \\
\hline \multirow{30}{*}{$\begin{array}{l}\text { Transferencia de } \\
\text { conocimiento }\end{array}$} & \multirow{14}{*}{$\begin{array}{l}\text { Recursos y } \\
\text { actividades }\end{array}$} & Contratos y convenios & 420 & 24,7 & 10,5 \\
\hline & & Emprendimiento & 203 & 12,0 & 5,1 \\
\hline & & Prácticas en empresa & 176 & 10,4 & 4,4 \\
\hline & & Captación, búsqueda de recursos y fondos & 155 & 9,1 & 3,9 \\
\hline & & Parque científico & 151 & 8,9 & 3,8 \\
\hline & & Consejo Social & 140 & 8,2 & 3,5 \\
\hline & & Desarrollo tecnológico & 120 & 7,1 & 3,0 \\
\hline & & Divulgación científica & 98 & 5,8 & 2,5 \\
\hline & & Intercambio de profesionales & 58 & 3,4 & 1,5 \\
\hline & & Consultoria, asesoramiento & 49 & 2,9 & 1,2 \\
\hline & & OTRI & 48 & 2,8 & 1,2 \\
\hline & & Cátedras de empresa & 31 & 1,8 & 0,8 \\
\hline & & Consorcios & 26 & 1,5 & 0,7 \\
\hline & & Incubadora de empresa & 22 & 1,3 & 0,6 \\
\hline & \multicolumn{2}{|c|}{ Total recursos y actividades } & 1.697 & 100,0 & 42,5 \\
\hline & \multirow{14}{*}{$\begin{array}{l}\text { Resultados } \\
\text { e impactos }\end{array}$} & Innovación & 524 & 22,8 & 13,1 \\
\hline & & Implicación, compromiso social & 463 & 20,2 & 11,6 \\
\hline & & Relación con entorno empresarial y productivo & 369 & 16,1 & 9,2 \\
\hline & & Transferencia tecnológica & 290 & 12,6 & 7,3 \\
\hline & & Servicio a la sociedad & 235 & 10,2 & 5,9 \\
\hline & & Creación de empresas & 124 & 5,4 & 3,1 \\
\hline & & Patentes & 89 & 3,9 & 2,2 \\
\hline & & Explotación comercial, comercialización & 68 & 3,0 & 1,7 \\
\hline & & Spin-off & 39 & 1,7 & 1,0 \\
\hline & & Diálogo social & 30 & 1,3 & 0,8 \\
\hline & & Utilidad social & 27 & 1,2 & 0,7 \\
\hline & & Licencias & 25 & 1,1 & 0,6 \\
\hline & & Start-up & 6 & 0,3 & 0,2 \\
\hline & & Modelos de utilidad & 6 & 0,3 & 0,2 \\
\hline & \multicolumn{2}{|c|}{ Total resultados e impactos } & 2.295 & 100,0 & 57,5 \\
\hline \multicolumn{3}{|c|}{ Total Transferencia de Conocimiento } & 3.992 & 100,0 & 100,0 \\
\hline
\end{tabular}

Fuente: Elaboración propia. 
$(26,7 \%)$, seguida de la creación, producción, generación de conocimiento $(22,3 \%)$ y las publicaciones $(16,2 \%)$. Se puede decir que para el conjunto de esta unidad de registro, los planes estratégicos hacen referencia a indicadores relacionados con el personal, con los proyectos y colaboraciones realizadas, con el número de publicaciones y con la política de evaluación de la investigación, no dando tanta importancia a los medios materiales y a la organización y ejecución de esta misión.

Finalmente, la unidad de registro "Transferencia de conocimiento" muestra para la dimensión "Recursos y Actividades" que los indicadores más frecuentes son los contratos y convenios $(24,7 \%)$, el emprendimiento $(12,0 \%)$, las prácticas en empresa $(10,4 \%)$ y la captación, búsqueda de recursos y fondos $(9,1 \%)$. Para la dimensión "Resultados e Impactos" el indicador más frecuente es el de innovación $(22,8 \%)$, seguida por la implicación, compromiso social $(20,2 \%)$ y la relación con el entorno empresarial y productivo $(16,1 \%)$. Así, se puede afirmar que, para el conjunto de esta unidad de registro, se da mayor relevancia a los indicadores relacionados con los logros que conlleva el desarrollo de la transferencia de conocimiento, como son la importancia de la innovación, su implicación social y todas aquellas actividades pertenecientes a la relación con el entorno empresarial y productivo. Sin embargo, resulta curioso que, dando tanta importancia a la innovación, no se le dé a aquellos medios que sirven para proteger industrialmente algunos resultados científicos, como son las patentes y los modelos de utilidad, ni a su transferencia efectiva mediante licencias; si bien, cabe pensar que este objetivo se encuentra recogido en el indicador más genérico de transferencia tecnológica. Asimismo, los planes estratégicos tampoco hacen especial hincapié en la creación de empresas spin-off y start-up.

\subsection{Visión comparada de las misiones de la universidad pública española}

A continuación, se presenta una comparativa de las misiones que manifiesta desarrollar, o que se propone desarrollar en el futuro, cada institución universitaria. Observando la distribución porcentual de los indicadores para cada unidad de registro, podemos ver las diferencias o similitudes entre las distintas universidades a la hora de planificar con mayor énfasis unas misiones $u$ otras, tratando de establecer un patrón que las identifique.

La Tabla 8 muestra la distribución porcentual de los indicadores contabilizados en cada plan estratégico (universidad), por dimensión y misión (unidad de registro). Los datos se presentan para el conjunto de unidades de contexto, es decir, considerando el global de la "Misión", la "Visión" y los "Ejes".

TABLA 8. DISTRIBUCIÓN DE LOS INDICADORES POR UNIVERSIDAD, DIMENSIÓN Y UNIDAD DE REGISTRO (FILA=100\%)

\begin{tabular}{|l|c|c|c|c|c|c|c|c|c|}
\hline \multirow{2}{*}{ Universidades } & \multicolumn{3}{c|}{ Recursos/Actividades } & \multicolumn{3}{c|}{ Resultados/Impactos } & \multicolumn{4}{c|}{ Subtotales } \\
\cline { 2 - 11 } & D & I & TC & D & I & TC & D & I & TC \\
\hline A Coruña & 25,5 & 19,3 & 9,0 & 24,8 & 11,7 & 9,7 & 50,3 & 31,0 & 18,7 \\
\hline Alicante & 38,9 & 10,6 & 8,1 & 21,2 & 11,1 & 10,1 & 60,1 & 21,7 & 18,2 \\
\hline Almeria & 42,3 & 13,9 & 12,1 & 17,1 & 6,2 & 8,4 & 59,4 & 20,1 & 20,5 \\
\hline Autónoma de Madrid & 42,2 & 17,7 & 7,5 & 14,5 & 6,5 & 11,7 & 56,7 & 24,2 & 19,2 \\
\hline Barcelona & 30,2 & 26,5 & 5,9 & 14,3 & 6,5 & 16,6 & 44,5 & 33,0 & 22,5 \\
\hline Burgos & 33,2 & 18,7 & 13,7 & 17,2 & 6,1 & 11,1 & 50,4 & 24,8 & 24,8 \\
\hline Cádiz & 26,5 & 14,1 & 15,7 & 18,2 & 6,6 & 18,9 & 44,7 & 20,7 & 34,6 \\
\hline Córdoba & 31,3 & 20,1 & 8,5 & 20,3 & 6,4 & 13,3 & 51,6 & 26,5 & 21,8 \\
\hline Girona & 27,9 & 21,6 & 10,3 & 16,3 & 8,9 & 15,0 & 44,2 & 30,5 & 25,3 \\
\hline Granada & 33,4 & 19,3 & 10,9 & 16,1 & 8,6 & 11,7 & 49,5 & 27,9 & 22,6 \\
\hline
\end{tabular}


TABLA 8. DISTRIBUCIÓN DE LOS INDICADORES POR UNIVERSIDAD, DIMENSIÓN Y UNIDAD DE REGISTRO (FILA=100\%) (continuación)

\begin{tabular}{|c|c|c|c|c|c|c|c|c|c|}
\hline \multirow{2}{*}{ Universidades } & \multicolumn{3}{|c|}{ Recursos/Actividades } & \multicolumn{3}{|c|}{ Resultados/Impactos } & \multicolumn{3}{|c|}{ Subtotales } \\
\hline & D & I & TC & D & I & TC & D & I & TC \\
\hline Huelva & 35,9 & 20,4 & 10,6 & 11,5 & 7,6 & 14,1 & 47,4 & 28,0 & 24,7 \\
\hline Islas Baleares & 29,0 & 13,1 & 8,2 & 20,6 & 11,6 & 17,5 & 49,6 & 24,7 & 25,7 \\
\hline Jaén & 37,6 & 17,1 & 11,8 & 15,2 & 8,7 & 9,6 & 52,8 & 25,8 & 21,4 \\
\hline Jaume I & 40,7 & 19,0 & 6,8 & 10,4 & 6,8 & 16,3 & 51,1 & 25,8 & 23,1 \\
\hline La Laguna & 35,8 & 25,9 & 10,7 & 12,5 & 4,4 & 10,6 & 48,3 & 30,3 & 21,3 \\
\hline La Rioja & 63,8 & 7,6 & 5,5 & 11,1 & 5,6 & 6,3 & 74,9 & 13,2 & 11,8 \\
\hline Las Palmas de Gran Canarias & 40,1 & 19,2 & 9,4 & 10,0 & 9,7 & 11,6 & 50,1 & 28,9 & 21,0 \\
\hline León & 35,2 & 18,5 & 10,3 & 20,6 & 6,0 & 9,4 & 55,8 & 24,5 & 19,7 \\
\hline Lleida & 32,9 & 16,6 & 13,3 & 15,7 & 4,8 & 16,6 & 48,6 & 21,4 & 29,9 \\
\hline Málaga & 29,5 & 20,4 & 13,0 & 13,4 & 6,9 & 16,7 & 42,9 & 27,3 & 29,7 \\
\hline Miguel Hernández & 33,2 & 18,7 & 13,7 & 17,2 & 6,1 & 11,1 & 50,4 & 24,8 & 24,8 \\
\hline Murcia & 30,4 & 24,4 & 7,9 & 12,6 & 9,2 & 15,6 & 43,0 & 33,6 & 23,5 \\
\hline Oviedo & 40,2 & 15,9 & 4,7 & 16,8 & 6,6 & 15,7 & 57,0 & 22,5 & 20,4 \\
\hline Pablo de Olavide & 38,6 & 20,3 & 9,3 & 14,4 & 6,9 & 10,5 & 53,0 & 27,2 & 19,8 \\
\hline País Vasco & 31,3 & 21,8 & 8,1 & 13,0 & 13,4 & 12,3 & 44,3 & 35,2 & 20,4 \\
\hline Politécnica de Catalunya & 41,6 & 11,4 & 12,9 & 13,9 & 6,7 & 13,6 & 55,5 & 18,1 & 26,5 \\
\hline Politécnica de Valencia & 22,4 & 17,1 & 9,3 & 22,0 & 12,0 & 17,1 & 44,4 & 29,1 & 26,4 \\
\hline Pública de Navarra & 29,8 & 21,0 & 8,5 & 20,6 & 6,6 & 13,5 & 50,4 & 27,6 & 22,0 \\
\hline Rovira i Virgili & 24,3 & 14,8 & 11,1 & 18,3 & 8,4 & 23,0 & 42,6 & 23,2 & 34,1 \\
\hline Salamanca & 29,3 & 9,8 & 7,3 & 20,7 & 14,6 & 18,3 & 50,0 & 24,4 & 25,6 \\
\hline Santiago de Compostela & 41,5 & 31,6 & 8,3 & 8,7 & 5,1 & 4,7 & 50,2 & 36,7 & 13,0 \\
\hline Sevilla & 33,4 & 19,3 & 10,9 & 16,1 & 8,6 & 11,7 & 49,5 & 27,9 & 22,6 \\
\hline Valencia & 39,6 & 15,7 & 8,9 & 14,8 & 8,9 & 12,1 & 54,4 & 24,6 & 21,0 \\
\hline Valladolid & 41,7 & 18,3 & 13,1 & 12,6 & 7,1 & 7,1 & 54,3 & 25,4 & 20,2 \\
\hline Vigo & 25,5 & 19,3 & 9,0 & 24,8 & 11,7 & 9,7 & 50,3 & 31,0 & 18,7 \\
\hline Zaragoza & 45,3 & 13,2 & 10,5 & 12,0 & 4,6 & 14,5 & 57,3 & 17,8 & 25,0 \\
\hline Total & 36,3 & 18,2 & 9,7 & 15,2 & 7,6 & 13,1 & 51,5 & 25,8 & 22,8 \\
\hline
\end{tabular}

Fuente: Elaboración propia.

Como se puede observar, la "Docencia" es la unidad de registro donde aparecen mayor porcentaje de indicadores $(51,5 \%)$ (véase Tabla 8), no sólo en el conjunto de las universidades analizadas, sino en todos y cada uno de los 36 planes estratégicos revisados.
Considerando los resultados por universidades, la Universidad de La Rioja es la que mayor énfasis pone en la "Docencia" ya que cerca del 75\% de los indicadores identificados en su plan hacen referencia a esta misión. Le sigue la Universidad de Alicante que le dedica el 60,1\% de los 
indicadores. El resto de instituciones tienen porcentajes próximos al $50 \%$, si bien oscilan entre $+/-10$ puntos, por lo que entre el $40 \%$ y el $60 \%$ de los indicadores, dependiendo de la universidad, están orientados a la "Docencia".

La "Investigación" es la segunda misión con mayor frecuencia de indicadores (25,8\%). De hecho, 25 de los 36 planes estratégicos analizados recogen en esta unidad los porcentajes más elevados después de la "Docencia". La universidad que recoge mayor proporción de indicadores en "Investigación" es la Universidad Santiago de Compostela $(36,5 \%)$ y la que menos es la Universidad de La Rioja $(13,2 \%)$. Podríamos decir, por tanto, que en segundo lugar, las universidades españolas están orientadas a la "Investigación", pues entre el $25 \%$ y el $35 \%$ de los indicadores aparecidos en los planes estratégicos corresponden a esta misión.

En cuanto a la "Transferencia de conocimiento", en general, los indicadores contabilizados vienen a representar entre el $15 \%$ y el $25 \%$ de los indicadores contabilizados en los planes estratégicos, como se ha dicho, por debajo de la "Investigación". Dos instituciones distribuyen en la misma proporción los indicadores contabilizados en "Investigación" y en "Transferencia de conocimiento", éstas son la Universidad de Burgos y la Universidad Miguel Hernández de Elche. Por su parte, nueve planes estratégicos muestran porcentajes de indicadores de "Transferencia de Conocimiento" superiores a la "Investigación". Éstas son la Universidad de Cádiz, la Universidad Rovira i Virgili, la Universidad de Lleida, la Universidad de Málaga, la Universidad Politécnica de Catalunya, la Universidad de las Islas Baleares, la Universidad de Salamanca, la Universidad de Zaragoza y la Universidad de Almería cuyos porcentajes se sitúan próximos al $30 \%$, superando esta cifra alguna de ellas.

Así pues, en base a estos resultados, cabe la posibilidad de que se pueda establecer una tipología de universidades públicas españolas, dado que, por un lado, encontramos universidades principalmente centradas en la docencia, y por otro lado, instituciones que prestan una mayor atención a la investigación y/o a la transferencia de conocimiento.

Con el objetivo de testar esta percepción resultante de los datos descriptivos obtenidos en el análisis de contenido, se aplica el análisis factorial. De su aplicación se obtienen dos factores totalmente independientes y ortogonales: uno de ellos relativo a la misión de docencia y el otro factor agrupa las dimensiones investigación y transferencia de conocimiento. Dichos factores explican el 99,8\% de la varianza total.

La agrupación resultante del análisis factorial hace pensar que, siguiendo el análisis de los planes estratégicos, las universidades públicas españolas consideran que sólo se transfiere el conocimiento que procede de la investigación, lo cual supone cierta desvinculación de estas dos misiones de la primera, la docencia, como si fueran compartimentos estancos. La literatura reciente está proporcionando enfoques mucho más integradores de las tres misiones (véase Laredo, 2007); sin embargo, parece que en el contexto español las universidades se encuentran alejadas de esta corriente.

En la Figura 2, se puede observar la agrupación de las universidades en función de su puntuación en cada uno de los factores. Así, las universidades situadas por debajo de la diagonal son universidades más orientadas hacia la misión de docencia (p.ej., Universidad de La Rioja, ya citada). En la parte superior de la diagonal encontramos las universidades preferentemente orientadas a la investigación y la transferencia de conocimiento (p.ej., Universidad Santiago de Compostela). Por último, las instituciones que se encuentran cerca de la diagonal presentan un equilibrio entre la Docencia y la Investigación junto a la Transferencia de Conocimiento.

\section{Conclusiones}

En el presente trabajo hemos utilizado el enfoque del Neoinstitucionalismo como base teórica explicativa de las tendencias de cambio en el campo organizativo de la educación universitaria pública en España. Concretamente, hemos analizado cuáles son las misiones que las universidades públicas españolas asumen de forma institucional. Para tal fin, la revisión realizada de los planes estratégicos de las universidades públicas españolas presenciales ha permitido enriquecer la visión institucional de estas organizaciones, ya que en dichos documentos se legitiman los valores y las misiones que promueven el cambio institucional. El método utilizado ha sido el análisis de contenido.

ARBOR Vol. 188753 enero-febrero [2012] 171-192 ISSN: 0210-1963 


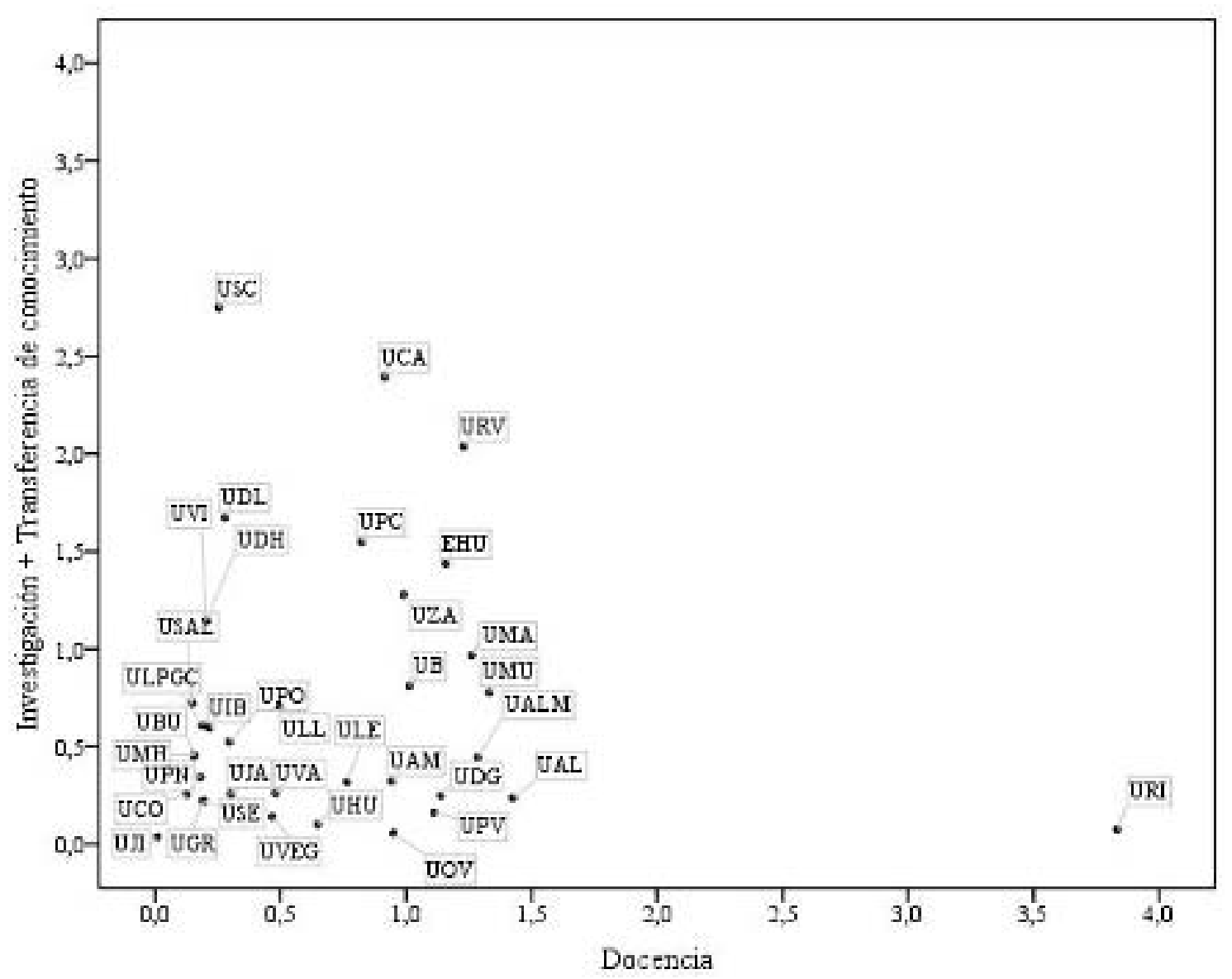

Fuente: Elaboración propia.

Los planes estratégicos revisados ponen énfasis en la misión "Docencia", ya que en esta unidad de registro se contabilizan más de la mitad de los indicadores. En segundo lugar, aparece la misión "Investigación", que registra algo más de un cuarto de los indicadores computados, y el resto aparecen registrados en la misión "Transferencia de conocimiento". El interés y la atención en la "Docencia" y la "Investigación" se centran principalmente en los "Recursos y Actividades", mientras que en la "Transferencia de conocimiento" se presta mayor atención a los "Resultados e Impactos" alcanzados.

En base a este resultado global, se podria pensar que la puesta en marcha de planes estratégicos en las universi- dades está funcionando como un mecanismo mimético y normativo que insta a las instituciones a fortalecer más unas misiones que otras para alcanzar el cambio organizacional. Sin embargo, como hemos visto en el análisis comparado, todas las universidades no presentan el mismo grado de institucionalización de cada misión. De hecho, las universidades pueden agruparse en dos tipos, en función del peso que han otorgado a cada misión en sus planes estratégicos; el primero enfocado prioritariamente a la docencia y el segundo a la investigación y la transferencia de conocimiento.

En este sentido, aunque el marco de análisis utilizado informa del proceso de reconfiguración de las universida- 
des, surge la necesidad de profundizar más en el estudio de las causas que influyen en ese proceso y plantearse si las universidades públicas españolas están cambiando sus patrones de comportamiento a tenor de algunos de los resultados encontrados a nivel institucional. Por ejemplo, la Universidad de Salamanca que, considerada como la más antigua de las universidades hispanas, ha estado caracterizada por su orientación a la enseñanza y la capacidad para atraer estudiantes de fuera de su región. Sin embargo, los resultados de este estudio ponen de manifiesto que, en sus planes estratégicos, si bien se hace alusión a la docencia como misión primaria, ésta es inferior a la media del Sistema Universitario Público Español (SUPE), dedicando más atención a la transferencia de conocimiento $y$, posteriormente, a la investigación. Ocurre algo parecido en la Universidad Politécnica de Catalunya pero, en este caso, en sentido inverso. Resulta llamativo que se obtengan porcentajes en la misión "Docencia" superiores al global del SUPE cuando se trata de una universidad eminentemente técnica $y$, por el contrario, aunque se sitúa bastante por encima de la media en la transferencia de conocimiento, se encuentra por debajo en la "Investigación". Estos resultados podrían reflejar que las instituciones tienden a reforzar en sus planes estratégicos sus debilidades y no tanto a potenciar sus fortalezas.

Además, podemos decir que no todas las universidades públicas españolas asumen institucionalmente por igual las tres misiones básicas de docencia, investigación y transferencia de conocimiento. La existencia de diferentes orientaciones estratégicas puede tener diversas implicaciones, especialmente en lo referente a las políticas universitarias y científicas, pues esa diversidad casa mal con la tendencia a realizar evaluaciones de desempeño homogéneas para todas las universidades, con el impacto que éstas pueden tener en los mecanismos de financiación de las universidades.

Por otra parte, convendría constatar si existe una relación entre lo que las universidades dicen que hacen en sus planes estratégicos y lo que realmente hacen mediante el uso de datos secundarios. Esta comprobación permitiría, además, conocer las relaciones existentes entre el desarrollo de las diferentes misiones de la universidad para evidenciar si existen relaciones de complementariedad o sustitución entre ellas.

\section{BIBLIOGRAFÍA}

Álamo Vera, F. R. y García Soto, M. G. (2007): "El proceso estratégico en el sector público: análisis en el contexto de las universidades españolas", Investigaciones Europeas, vol. 113, n. 2 , pp. 113-129.

Arias Coello, A. y Simón Martín, J. (2005): "Planes estratégicos en las bibliotecas universitarias españolas", Documentación de las Ciencias de la Información, vol. 28, pp. 73-85.

Bhattacharjee, M. (2008): "Strategic Management in Higher Educational Institutions: Emphasis on Career Orientation in Management and Technical Institutes", The Icfai University Journal of Higher Education, vol. 3, n. ${ }^{3}$, pp. 38-47.
Campbell, J. L. (2004): "Problems of institutional analysis", en J. L. Campbell, Institutional change and globalization, Princeton, Princeton University Press, pp. 1-30.

Colyvas, J. A. y Powell, W. W. (2006): "Roads to institutionalization: the remaking of boundaries between public and private science", Research in Organizational Behavior, vol. 27, pp. 305-353.

David, P. A. y Foray, D. (1994): Accessing and Expanding the Science and Technology Knowledge-base [en linea], Working Group on Innovation and Technology Policy, DSTI/STP/ TIP 94(4), OCDE, Paris, [ref. 4 de mayo de 2011]. Disponible en Web: http://www.oecd.org/dataoecd/34/ 15/2097997.pdf.
Aceptado: 17 de junio de 2011 
Demeritt, D. (2000): "The new social contract for science: accountability, relevance and value in US and UK science and research policy", Antipode, vol. 32, n. 3, pp. 308-329.

DiMaggio, P. J. y Powell, W. W. (1983): "The iron cage revisited: institutional isomorphism and collective rationality in organizational fields", American Sociological Review, vol. 48, n. ${ }^{\circ} 2$, pp. 147-160.

Frølich, N. y Klitkou, A. (2006): "Strategic management of higher education institutions: performance funding and research output", en Actas de la Conference on Indicators on Science, Technology and Innovation, Special session on Indicators for strategic management of higher education institutions (Lugano, 15-17 de noviembre de 2006), pp. 1-20.

Fuentes Romero, J. J. (2001): "Planificación de centros bibliotecarios", Revista General de Información y Documentación, vol. 11, n. ${ }^{\circ}$ 1, pp. 49-82.

Geuna, A. (1996): European universities: an interpretative history [en línea], Research memoranda, 8, MERIT, Maastricht [ref. de 4 de mayo de 2011]. Disponible en Web: http://edocs. ub.unimaas.nl/loader/file.asp?id=56.

Godin, B. y Gingras, Y. (2000): "The place of universities in the system of knowledge production", Research Policy, vol. 29, pp. 273-278.

Göransson, B.; Maharajh, R. y Schmoch, U. (2009): "New activities of universities in transfer and extension: multiple requirements and manifold Solutions", Science and Public Policy, vol. 36, n. ${ }^{\circ}$, pp. 157-164.

Hawley, A. (1968): "Human ecology", en D. L. Sills (ed.), International enciclo- pedia of the social sciences, Nueva York, Macmillam, pp. 328-337.

Kerr, C. (1963): The uses of the University, Cambridge, Harvard College.

Laredo, P. (2007): "Revisiting the Third Mission of Universities: Toward a Renewed Categorization of University Activities?" Higher Education Policy, 20, pp. 441-456.

Martín, C. J. (2000): "La misión de la universidad en el S.XXI: ¿Torre de marfil, parque de diversión, oficina comercial o pensar lo impensable?", Estudios del Hombre, vol. 12, pp. 43-59.

Meyer, J. (1977): "The effects of education as an institution", American Journal of Sociology, vol. 63, pp. 55-77.

Meyer, J. (2002): "From loose coupling to tight management? Making sense of the changing landscape in organization and management". Journal of Education Administration, vol. 40, n. ${ }^{\circ}$, pp. 515-520.

Meyer, J. (2006): "The rise and decline of the common school as an institution: taking "myth and ceremony" seriously", en J. Meyer y B. Rowan (eds.), The new institutionalism in education, Nueva York, State University of New York Press, pp. 51-66.

Meyer, J. y Rowan, B. (1977): "Institutionalized organizations: formal structure as myth and ceremony", American Journal of Sociology, vol. 83, n. 2 , pp. 340-363.

Meyer, J. y Rowan, B. (2006): The new institutionalism in education, Nueva York, State University of New York Press.

Ministerio de Ciencia e Innovación (2010): Anteproyecto de Ley de la Ciencia, la Tecnología y la Innovación [ref. 4 de mayo de 2011]. Disponible en Web: http://www.micinn.es/stfls/MICINN/
Ministerio/FICHEROS/Ley_ciencia_ tecnolog_e_innovac_Borrador_UNO_ Hecho_publico_V_22_02_10.pdf.

Mintzberg, H.; Quinn, J. B. y Voyer, J. (1997): El proceso estratégico. Concepto, contextos y casos, Prentice Hall, México.

Molas-Gallart, J.; Salter, A.; Patel, P.; Scott, A. y Duran, X. (2002): Measuring Third Stream Activities. Final Report to the Russell Group of Universities, Science and Technology Policy Research (SPRU), University of Sussex. Freeman Centre, Falmer, Brighton, East Sussex, BN1 90E, United Kingdom.

OCDE (1999): University research in transition, OCDE, Paris.

Sánchez, M. P. y Elena, S. (2007): "La gestión estratégica de la universidad contemporánea: reflexiones sobre la potencialidad de los modelos de capital intelectual", Revista madritd, vol. 42.

Scott, P. (1998): "Fisuras en la Torre de marfil", El correo de la Unesco, septiembre, pp. 18-20.

Taylor, J. y Machado, M. L. (2006): "Higher education leadership and management: from conflict to interdependence through strategic planning", Tertiary Education and Management, vol. 12, pp. 137-160.

Torres, P.; Villafán, J. y Álvarez, M. L. (2008): "Planeación estratégica y desarrollo organizacional en instituciones educativas: el estudio de un caso universitario en México", Revista Iberoamericana de Educación, vol. 47, n. ${ }^{\circ}$, pp. 1-11.

Vilalta, J. M. (2001): "Implantación y evaluación de planes estratégicos en las universidades", XIX Jornadas de Gerencia Universitaria, Universidad $\mathrm{Pa}$ blo de Olavide, Sevilla, octubre. 